Pure Appl. Chem., Vol. 76, No. 7-8, pp. 1321-1335, 2004.

(C) 2004 IUPAC

\title{
Polymeric micelles for oral drug delivery: Why and how*
}

\author{
Mira F. Francis ${ }^{1}$, Mariana Cristea ${ }^{1,3}$, and Françoise M. Winnik ${ }^{1,2, \ddagger}$ \\ ${ }^{1}$ Faculty of Pharmacy, University of Montreal, C.P. 6128 Succ. Centre-ville, \\ Montreal, Quebec H3C 3J7, Canada; ${ }^{2}$ Department of Chemistry, University of \\ Montreal, C.P. 6128 Succ. Centre-ville, Montreal, Quebec H3C 3J7, Canada; \\ ${ }^{3}$ Petru Poni Institute of Macromolecular Chemistry, lasi 6600, Romania
}

\begin{abstract}
The oral delivery of drugs is regarded as the optimal means for achieving therapeutic effects owing to increased patient compliance. Unfortunately, the oral delivery route is beset with problems such as gastrointestinal (GI) destruction of labile molecules, low levels of macromolecular absorption, etc. To reduce the impact of digestive enzymes and to ensure the absorption of bioactive agents in an unaltered form, molecules may be incorporated into microparticulate carriers. Many approaches to achieve the oral absorption of a wide variety of drugs are currently under investigation. Among the different polymer-based drug delivery systems, polymeric micelles represent a promising delivery vehicle especially intended for poorly water-soluble pharmaceutical active ingredients in order to improve their oral bioavailability. Recent findings of a dextran-based polymeric micelle study for solubilization of a highly lipophilic drug, cyclosporin A (CsA), will be discussed.
\end{abstract}

\section{INTRODUCTION}

Oral administration of therapeutic agents represents by far the easiest and most convenient route of drug delivery, especially in the case of chronic therapies [1]. While convenient from the patient's perspective, oral drug formulation presents a challenge to the chemist, who has to design delivery systems optimizing drug stability in the gastrointestinal (GI) tract, such that a desirable pharmacokinetic profile may be attained for a given drug [2]. Indeed, the GI tract presents a variety of hurdles for a drug, from morphological barriers (mucus layer, microvilli, etc.) to stringent physiological factors (a wide range of $\mathrm{pH}$, enzymatic activities, specific transport mechanisms, etc.), which all conspire to limit intestinal absorption of drug. In the case of poorly water-soluble drugs, the dissolution time in the GI contents may be longer than the transit time to the intended absorptive sites [3]. Therefore, dissolution of drugs is quite often the rate-limiting step which, ultimately, controls the bioavailability of the drug [4]. This poses a major challenge for effective delivery of poorly water-soluble therapeutics via the oral route [5].

One approach to enhance the absorption efficiency and bioavailability of highly lipophilic drugs taken orally consists in using a particulate delivery system soluble or dispersible in an aqueous environment. This strategy is based on the following premises: particulate systems (a) provide the drug with some level of protection against degradation within the GI tract; (b) prolong the drug transit time and facilitate translocation of the drug across epithelial barriers, thus improving drug absorption; and (c) may be targeted to specific sites and carry functionalities that assist specific absorption pathways.

\footnotetext{
*Lecture presented at the symposium "Controlling the self assembly in macromolecular systems: From nature to chemistry to functional properties", as part of the $39^{\text {th }}$ IUPAC Congress and $86^{\text {th }}$ Conference of the Canadian Society for Chemistry: Chemistry at the Interfaces, Ottawa, Canada, 10-15 August 2003. Other Congress presentations are published in this issue, pp. 1295-1603.

${ }^{\ddagger}$ Corresponding author: Tel.: 1-514-340-5179; Fax: 1-514-340-3245; E-mail: francoise.winnik@ umontreal.ca
} 


\section{PHYSICOCHEMICAL CHARACTERISTICS OF ORAL DELIVERY SYSTEMS}

The transport of a drug across the intestinal membrane is a complex transfer process involving several mechanisms. Based on the physiology of the intestinal epithelium, three potential routes for drug transport across intestinal barriers have been considered (Fig. 1): (1) the paracellular route, a passive, diffusional transport pathway taken by small, hydrophilic molecules (e.g., mannitol) which can pass through the tight junction channels between adjacent epithelial cells; (2) transcellular passive diffusion of lipophilic molecules through the lipid bilayer and the membrane-bound protein regions of the cell membrane; and (3) transcellular receptor-mediated transcytosis of surface-bound ligands such as intrinsic factor-cobalamin complexes. The extent of particle uptake through the absorbing membrane varies considerably depending on its size, chemical composition, hydrophobicity, and charge.

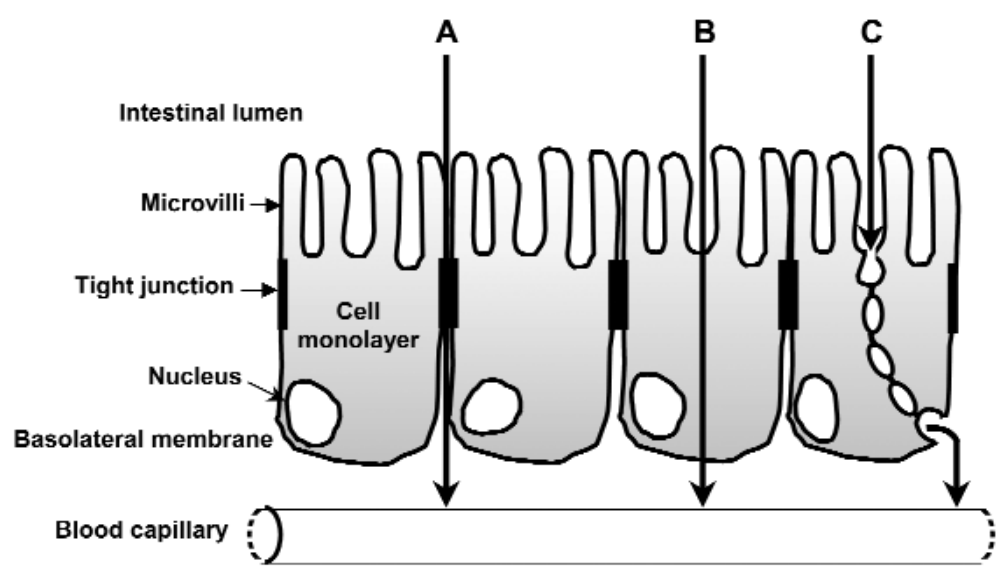

Fig. 1 Schematic representation of intestinal epithelial cells showing potential transepithelial pathways: (A) paracellular route; $(\mathbf{B})$ transcellular passive diffusion; and (C) transcellular receptor-mediated transcytosis.

- $\quad$ Particle size: the uptake of particles within the intestine and the extent of drug absorption increase with decreasing particle size and increasing specific surface area [6-8]. Jani et al. [9,10] assessed the size-dependence of the uptake of nanoparticles by the rat intestine by monitoring their appearance in the systemic circulation and their distribution in different tissues. After administration of equivalent doses, $33 \%$ of nanoparticles $50 \mathrm{~nm}$ in diameter and $26 \%$ of the $100-\mathrm{nm}$ nanoparticles were detected in the intestinal mucosa and gut-associated lymphoid tissues, whereas, in the case of 500-nm particles, only $10 \%$ were found in the intestinal tissues. The uptake of particles larger than $1 \mu \mathrm{m}$ in diameter was marginal. Moreover, these micron-sized particles were found only in the lymph nodes of the small intestine (Peyer's patches). Desai et al. [11] reported similar findings for the absorption characteristics of nanoparticles prepared from poly(lactide-co-glycolic acid) (PLGA). The uptake of 100-nm nanoparticles in the rat intestine was significantly increased compared to larger particles of 1 and $10 \mu \mathrm{m}$. Therefore, size is an important parameter controlling the internalization of nanoparticles into epithelia of the GI tract, and as a rule, sizes smaller than $500 \mathrm{~nm}$ are required.

- Chemical composition: A comparative study investigating a range of polymeric particles suggests that the extent of drug absorption depends on the hydrophobicity of the polymer [12]. It would appear that particles consisting of the hydrophobic polymer poly(styrene), are absorbed more readily than PLGA, a somewhat more hydrophilic polymer.

- Particle charge: Particle charge is also an important factor that determines the extent of uptake from the GI tract $[9,13]$. Carboxylated poly(styrene) nanoparticles show a significantly decreased affinity to intestinal epithelia, compared to positively charged and uncharged poly(styrene) 
nanoparticles [9]. However, Mathiowitz et al. [14] observed a highly increased adhesion of negatively charged poly(anhydride) copolymers to cell surfaces, resulting in an enhanced absorption rate of the encapsulated drug, compared to drug encapsulated in neutral particles. In their investigation of the affinity of negatively charged poly(acrylic acid) nanoparticles toward intestinal epithelia, Kriwet et al. [15] reached the same conclusion. Thus, neutral and positively charged nanoparticles have a higher affinity to intestinal epithelia than negatively charged nanoparticles, but negatively charged nanoparticles exhibit bioadhesive properties, which may also favor the transport process.

\section{APPROACHES}

Several particulate systems have been reported as effective carriers of therapeutic agents administered orally. Among them are liposomes, which have been used, for example, to administer insulin by the oral route [16]. Liposome formulations are particularly attractive, as they may carry lipid-soluble drugs in the lipid bilayer and, at the same time, water-soluble drugs in the aqueous core [17]. They are prepared routinely under mild conditions which minimize drug denaturation during encapsulation. Unfortunately, most liposome formulations are readily disrupted by intestinal detergents, such as bile salts, and are subject to degradation by intestinal phospholipases. Both mechanisms trigger the premature release of the liposome-entrapped drug in the GI fluid $[18,19]$. In addition to liposomes, other colloidal formulations, such as emulsions and microemulsions, are effective in the oral administration of drugs with limited solubility in biological fluids [20,21]. Microemulsions (oil/water) are bicontinuous systems composed of a continuous water phase containing nanometer-sized oil droplets separated by a surfactant/cosurfactant-rich interfacial region. Compared to emulsions, they offer several advantages as drug delivery systems, such as high solubilization capacity, thermodynamic stability, and ease of preparation [21]. In several cases, administration of microemulsions, especially formulations based on castor oil, has triggered adverse events, most frequently renal dysfunction, hypertension, and hepatotoxicity $[22,23]$. Recently, a new class of delivery system has been under intense scrutiny. They are nanoparticles ranging in size from $\sim 15$ to $150 \mathrm{~nm}$ [24,25] such as nanospheres [26], nanocapsules [16,27], hydrogels [28-30], and silica particles coated with proteins, labile drugs, and hormones. Matsuno et al. [31] found that nearly spherical poly(vinylpyrrolidone)-coated silica gel particles with an average diameter of $30 \mathrm{~nm}$ were taken up effectively by mouse enterocytes. Polymers investigated in nanocarrier formulations for oral drug delivery include poly(alkylcyanoacrylates) [27], and terpolymers of methyl methacrylate, 2-hydroxyethylmethacrylate, and $n$-butylacrylate [32]. The methodologies reviewed briefly in this section have met success in various therapies, and interested readers are referred to reviews on the subject [33-37]. The focus of this article is a yet different approach toward enhancing the bioavailability of highly lipophilic drugs, based on the molecular dissolution of a drug within the hydrophobic core of polymeric micelles.

\section{MICELLES: DEFINITION AND ADVANTAGES}

In the late 1960s, micelles drew much attention as drug carriers owing to their easily controlled properties and good pharmacological characteristics [38,39]. Micelles are formed when amphiphiles are placed in water. They consist of an inner core of assembled hydrophobic segments capable of solubilizing lipophilic substances and an outer hydrophilic corona serving as a stabilizing interface between the hydrophobic core and the external aqueous environment [40]. Depending on the delivery purpose, one can select the size, charge, and surface properties of these carriers simply by adding new ingredients to the mixture of amphiphilic substances before micelle preparation and/or by variation of the preparation method.

Micelles as drug carriers provide a set of advantages - they physically entrap sparingly soluble pharmaceuticals and deliver them to the desired site of action at concentrations that can exceed their in- 
trinsic water solubility and thus increase their bioavailability. The stability of the drug is also increased through micelle incorporation. Furthermore, undesirable side effects are lessened, as contact of the drug with inactivating species, such as enzymes present in biological fluids, are minimized, in comparison with free drug [41-43]. They can be prepared in large quantities easily and reproducibly [44,45]. By far the most important feature of micellar delivery systems, which distinguish them from other particulate drug carriers, lies in their small size ( 10 to $30 \mathrm{~nm})$ and the narrow size distribution [6].

Micelles made of nonionic surfactants are widely used as adjuvants and drug carrier systems in many areas of pharmaceutical technology and controlled drug delivery [46-51]. Such a high level of activity has brought a great deal of diversity to this field, since most groups introduced their own micelle system formed from unique hydrophilic-hydrophobic combinations. In almost all cases, the hydrophilic outer shell consists of poly(ethylene oxide) (PEO) chains, owing to their high degree of hydration and large excluded volume inducing repulsive forces, which contribute to the stabilization of the micelle [44,52-54]. In addition, the PEO corona prevents recognition by the reticuloendothelial system and therefore minimizes elimination of the micelle from the bloodstream. Thus, these so-called "stealth" properties of the PEO corona result in increased blood circulation times and allow drugs to be administered over prolonged periods of time [55-57]. A wide range of hydrophobic blocks have been explored, resulting in different micellar systems with distinct physicochemical properties.

Surfactant micelles form only above a critical concentration, the critical micelle concentration (CMC), and rapidly break apart upon dilution, which can result in premature leakage of the drug and its precipitation in situ. These limitations of surfactant micelles as drug delivery carriers triggered the search for micelles of significantly enhanced stability and solubilizing power.

The use of polymer-based micelles has gained much attention because of the high diversity of polymers, their biocompatibility, biodegradibility, and the multiplicity of functional groups they display for the conjugation of pilot molecules [30].

\section{POLYMERIC MICELLES}

Like their low-molecular-weight counterparts, amphiphilic polymers associate in water to form "polymeric micelles" [58], consisting of a hydrophobic core stabilized by a corona of hydrophilic polymeric chains exposed to the aqueous environment [59]. Polymeric micelles can be used as efficient carriers for compounds, which alone exhibit poor solubility, undesired pharmacokinetics, and low stability in a physiological environment. The hydrophilic shell contributes greatly to the pharmaceutical behavior of polymeric formulations by maintaining the micelles in a dispersed state, as well as by decreasing undesirable drug interactions with cells and proteins through steric-stabilization effects. The size of polymeric micelles ranges from $\sim 10$ to $\sim 100 \mathrm{~nm}$, and usually the size distribution is narrow [59]. This topology is similar to that of surfactant micelles, hence polymeric micelles can be expected to solubilize hydrophobic drugs within their core. However, there are significant differences between the two types of assemblies from the physicochemical viewpoint. The polymer concentration at which the association first takes place, sometimes known as the critical association concentration (CAC), is lower by several orders of magnitudes than typical surfactant CMC values. Thus, polymeric micelles are more stable toward dilution in biological fluids. They can increase drug bioavailability and retention, since the drug is well protected from possible inactivation under the effect of their biological surroundings [60].

Polymeric micelles have been studied extensively as delivery medium for injectable drug formulations of poorly water-soluble drugs such as paclitaxel, indomethacin, amphotericin B, adriamycin, and dihydrotestosterone. Overall, they proved to be highly effective drug delivery vehicles [61-65]. To date, most contributions in the area of polymeric micelles for oral formulations, have been made by the group of Kabanov [66-71]. Their work focused mostly on micelles formed from commercially available Pluronic ${ }^{\circledR}$ triblock copolymers [also termed Poloxamer; poly(ethylene oxide) ${ }_{x}-b$-poly(propylene oxide) $)_{y}-b$-poly(ethylene oxide) $\left.{ }_{x}, \mathrm{PEO}_{x}-b-\mathrm{PPO}_{y}-b-\mathrm{PEO}_{x}\right]$, and more recently, on block ionomer complexes as carriers for DNA [72]. 
Our approach to oral drug delivery through polymeric micelles was to design with care the chemical composition and architecture of the amphiphilic polymers forming the polymeric micelle vehicle. To maximize our chances of success, we chose to base the new materials on polysaccharides, which are nontoxic, naturally occurring polymers, and readily available in a range of molecular weights. We selected hydroxypropylcellulose (HPC), a nonionic water-soluble cellulose derivative, and dextran (DEX), a glucose polymer with predominantly 1,6- $\alpha$-glycosidic linkages [73]. Both polymers are nontoxic and nonirritant polysaccharides. They are used in medicine (e.g., DEX as plasma substitute both locally and systemically [74] and HPC in oral tablet formulations, where it acts as a disintegrant [75] and as a binder in granulation [76], due to its bioadhesive properties). In order to impart either DEX or HPC with an amphiphilic character, we linked to their backbone a small number of hydrophobic cetyl groups, as described below in the case of DEX. We chose to attach the hydrophobic groups to the polysaccharide hydrophilic backbone via a short PEO linker, yielding DEX grafted with $\mathrm{PEO}_{10}-\mathrm{C}_{16}$ (DEX- $g-\mathrm{PEO}_{10}-\mathrm{C}_{16}$ or HPC- $g$ - $\mathrm{PEO}_{10}-\mathrm{C}_{16}$ ); the digit next to PEO refers to the number of ethylene oxide groups in PEO, and the digit next to the carbon refers to the number of carbons in the alkyl chains (Fig. 2); and to exploit the solubilizing potential of this copolymer toward poorly water-soluble drugs. In aqueous solution, hydrophobically modified (HM) polysaccharides associate into polymeric micelles above the CAC. Thus, a lipophilic drug can be entrapped in the hydrophobic core formed by the alkyl residues, while the hydrophilic polysaccharide chains stabilize the system in the aqueous environment. In this article, we present the design, chemical characterization, and in vitro evaluation of HM DEX-based polymeric micelles as an example of the novel polymer-based nanocarriers for oral delivery of poorly water-soluble drugs developed recently in our group.

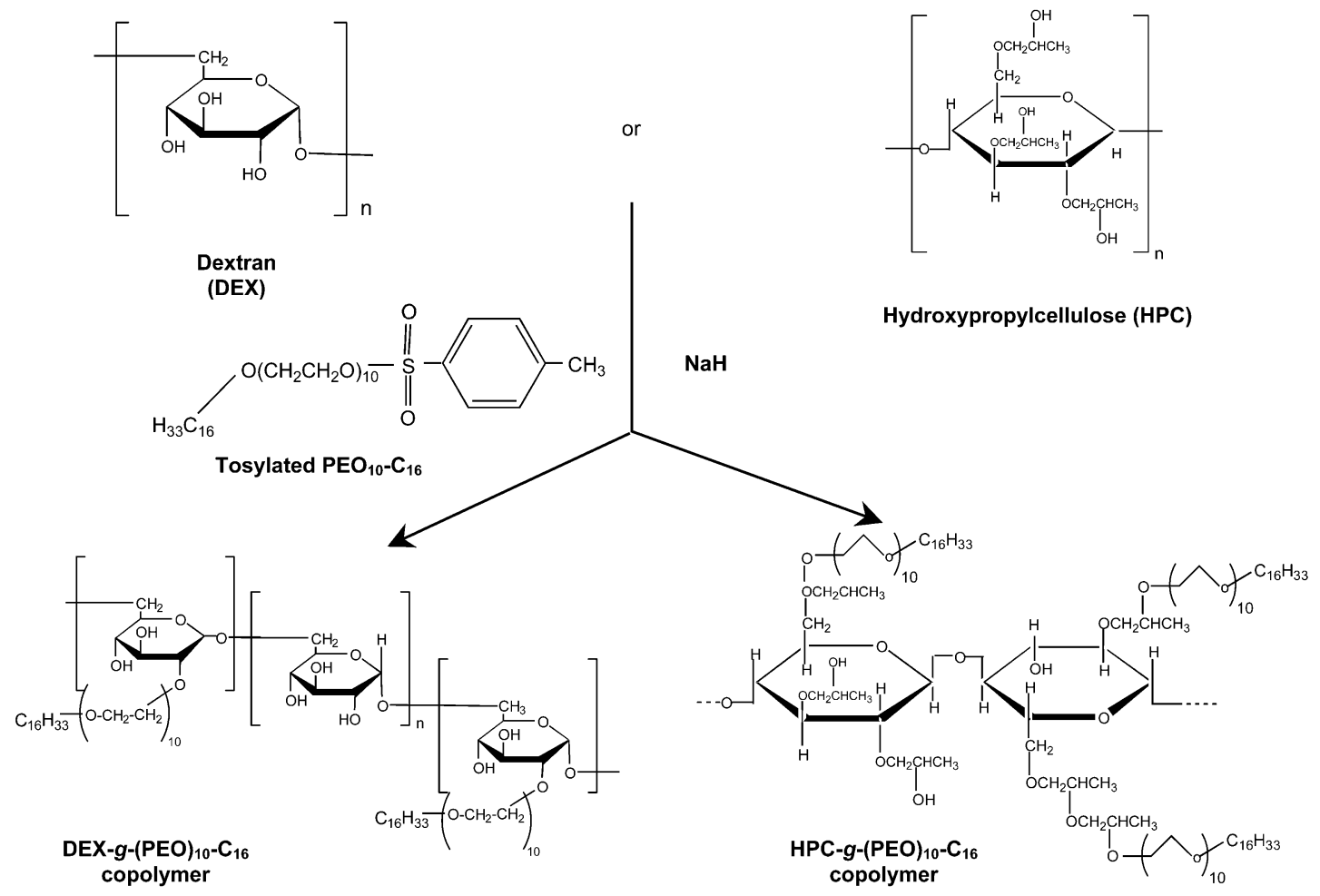

Fig. 2 Synthesis of HM-DEX and HM-hydroxypropylcellulose copolymers. 


\section{SYNTHESIS AND CHARACTERIZATION OF HM DEX COPOLYMERS}

HM DEX- $g-\mathrm{PEO}_{10}-\mathrm{C}_{16}$ graft copolymers were synthesized following the scheme shown in Fig. 2 [77]. A tosylated PEO (10) cetyl ether $\left(\mathrm{PEO}_{10} \mathrm{C}_{16}\right)$ was coupled to DEX T10 (DEX; MW $10000 \mathrm{Da}$ ) by a Williamson ether synthesis reaction. The resulting DEX-g-PEO ${ }_{10}-\mathrm{C}_{16}$ copolymers were purified by soxhlet extraction with dichloromethane to remove all free $\mathrm{PEO}_{10}-\mathrm{C}_{16}$. The extent of grafting (Table 1) was determined from ${ }^{1} \mathrm{H}$ NMR spectroscopy data, using the signal due to the resonance of the terminal cetyl methyl protons $(\delta \sim 0.85 \mathrm{ppm})$ and the signal due to the resonance of the anomeric protons of DEX $\left(\delta \sim 4.7 \mathrm{ppm}\right.$ ) (Fig. 3). Depending on the initial relative concentration of DEX and $\mathrm{PEO}_{10}-\mathrm{C}_{16}$, amphiphilic DEXs carrying from 2.3 to $15 \mathrm{~mol} \% \mathrm{PEO}_{10}-\mathrm{C}_{16}$ (relative to the number of glucose units) were prepared in excellent yield and a high level of purity.

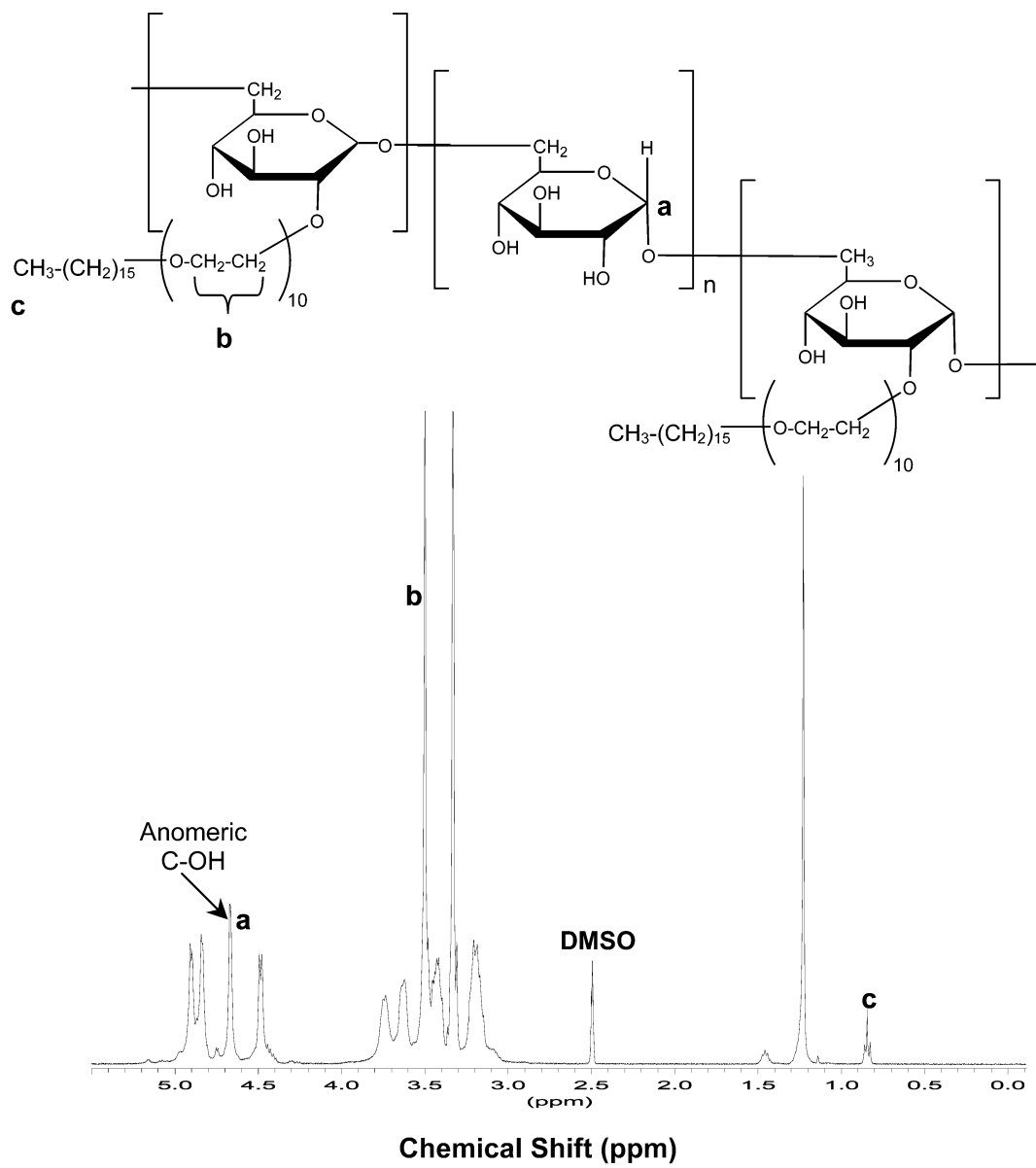

Fig. 3 Chemical structure of DEX- $g-\mathrm{PEO}_{10}-\mathrm{C}_{16}$ and its ${ }^{1} \mathrm{H}$ NMR spectrum in DMSO- $d_{6}$. 
Table 1 Physicochemical properties of DEX-g-PEO ${ }_{10}-\mathrm{C}_{16}$ polymeric micelles.

\begin{tabular}{|c|c|c|c|c|}
\hline $\begin{array}{l}\text { Polymer } \\
\text { composition }\end{array}$ & $\begin{array}{l}\text { Grafted PEO-C } \\
\quad(\mathrm{mol} \%)^{\mathrm{a}}\end{array}$ & $\begin{array}{l}\mathrm{CAC}^{\mathrm{b}} \\
(\mathrm{mg} / \mathrm{l})\end{array}$ & $\begin{array}{l}\text { Mean diameter } \\
(\mathrm{nm} \pm \mathrm{SD})\end{array}$ & $\begin{array}{c}\text { Maximum } \\
\text { CsA loading } \\
(\% \text { d } / w)\end{array}$ \\
\hline Dextran T10 & 0 & - & - & $0.6 \pm 0.1$ \\
\hline $\mathrm{PEO}_{10}-\mathrm{C}_{16}$ & - & $4.3 \pm 1$ & - & $17.5 \pm 0.5$ \\
\hline DEX- $g$-PEO $10-C_{16}$ & $2.3 \pm 0.1$ & $7.6 \pm 0.3$ & $44 \pm 4$ & $1.1 \pm 0.2$ \\
\hline DEX- $g-\mathrm{PEO}_{10}-\mathrm{C}_{16}$ & $6.4 \pm 0.1$ & $4.2 \pm 0.4$ & $12 \pm 3$ & $6.8 \pm 0.2$ \\
\hline 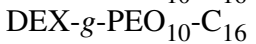 & $15.2 \pm 0.1$ & $3.7 \pm 0.2$ & $11 \pm 5$ & $8.5 \pm 0.6$ \\
\hline
\end{tabular}

${ }^{\mathrm{a}}$ Determined by ${ }^{1} \mathrm{H}$ NMR measurement.

${ }^{\mathrm{b}}$ Determined by change in $I_{336} / I_{333}$ ratio of pyrene fluorescence with log polymer concentration at $25{ }^{\circ} \mathrm{C}$.

${ }^{\mathrm{c}}$ Determined by DLS measurements at $25^{\circ} \mathrm{C}$ with a scattering angle of $90^{\circ}$.

${ }^{\mathrm{d}}$ Determined by HPLC analysis with UV detection at $210 \mathrm{~nm}$.

\section{PHYSICOCHEMICAL CHARACTERISTICS OF POLYMERIC MICELLES}

As discussed in the introduction, the ability of polymeric micelles to act as effective drug delivery systems is controlled, to a large extent, by their size and their CAC, defined as the copolymer concentration below which only single chains exist. In solutions of the copolymer of concentration higher than the CAC, micelles and single chains coexist. This definition is used commonly to describe polymeric micelles, in analogy with the CMC of surfactant micelles, although the mechanism of micellization may not be the same for amphiphilic polymers and surfactants [78,79]. A delivery system is subject to "sink conditions" or severe dilution in the GI fluid upon oral administration. It is important to know the CAC value in order to select dosage compositions such that the copolymer concentration remains above the CAC immediately upon administration. The CAC of amphiphilic copolymers is determined by many factors, such as the nature and length of the core-forming segments, and the length of the hydrophilic chain. Amphiphilic copolymers that contain highly hydrophobic residues have lower CAC values in water than those that include the less hydrophobic residues. For a series of copolymers, if the coronaforming chain is kept constant, an increase in the molecular weight of the core-forming segment will decrease the CAC [80]. To a lesser extent, if the length of the core-forming segment is maintained at a constant length, an increase in the length of the hydrophilic chain will cause an increase in the value of the CAC [81,82].

The CAC of polymeric micelles can be estimated by fluorescence spectroscopy using pyrene, a hydrophobic fluorescence probe that preferentially partitions into the hydrophobic core of the micelle. Pyrene undergoes changes in its photophysical properties as a result of the change in the micropolarity it experiences upon diffusion from bulk water (hydrophilic environment) into the micelle core (hydrophobic environment) $[83,84]$. Two methods exist for determining the CAC of polymeric micelles with pyrene fluorescence [85]. The original method, proposed by Kalyanasundaram et al. [86], takes advantage of the changes in the vibronic fine structure of the pyrene emission and monitors the changes in the ratio of the intensities $I_{1}$ and $I_{3}$ of the $[0,0]$ and $[0,2]$ bands, respectively. More recently, it has been suggested that a more accurate determination of the CAC can be obtained by monitoring the changes in the ratio of the pyrene excitation spectra intensities [87] at $\lambda=333 \mathrm{~nm}$ for pyrene in water and $\lambda=336$ $\mathrm{nm}$ for pyrene in a hydrophobic medium (Fig. 4). By plotting the $I_{336} / I_{333}$ intensity ratios vs. the logarithm of the concentration of the aqueous solutions of copolymer, sigmoidal curves are obtained, where, at the CAC, a sharp increase is observed in the fluorescence intensity ratio $\left(I_{336} / I_{333}\right)$ as the polymer concentration increases (Fig. 4).

We used the latter method to estimate the $\mathrm{CAC}$ of the DEX- $g-\mathrm{PEO}_{10}-\mathrm{C}_{16}$ copolymers. As shown in Table 1, the CAC values decrease with increasing molar content of $\mathrm{PEO}_{10}-\mathrm{C}_{16}$ residues linked to the 


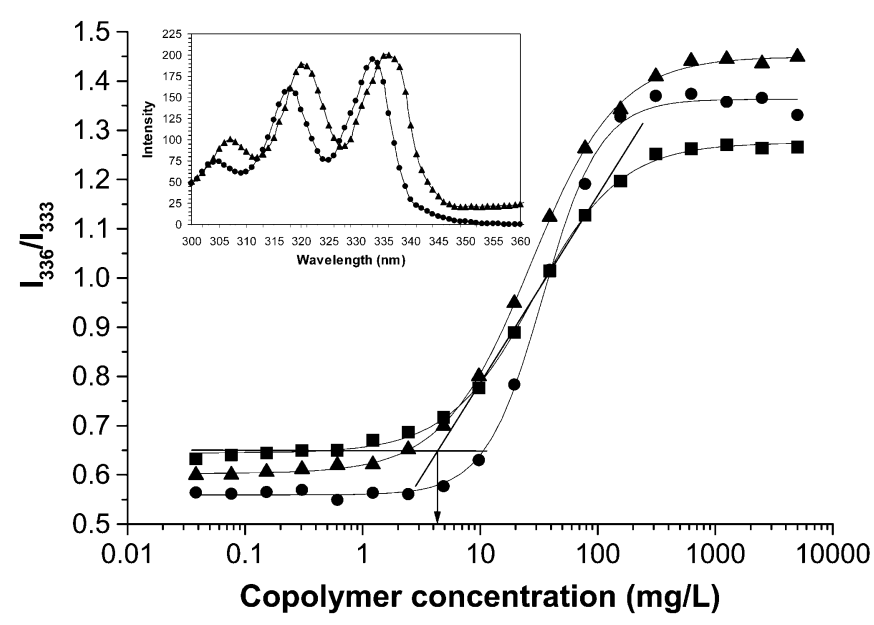

Fig. 4 Plot of the intensity ratio I336/I333 (from pyrene excitation spectra) as a function of concentration

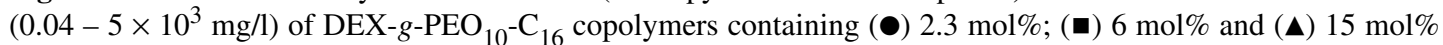
of grafted $\mathrm{PEO}_{10}-\mathrm{C}_{16}$ residues. Each value is the mean of two independent measurements. Inset: Excitation spectra of pyrene $\left(2 \times 10^{-7} \mathrm{M}\right.$ aqueous solution) monitored at $\lambda_{\text {em }} 390 \mathrm{~nm}$ in absence $(\bullet)$ or presence $(\boldsymbol{\Delta})$ of HM DEX copolymer.

DEX backbone. These results are consistent with recent evidence, suggesting that the increase in the length of a hydrophobic residue for a given length of a hydrophilic polymer chain causes noticeable decrease in CAC value and increase in micelle stability [78].

The size of micelles is controlled by the length of the core-forming segment and the length of the corona-forming chain $[88,89]$, but it depends also on the method of micellization selected for the preparation of micelles. For micelles produced using the dialysis method, it varies depending on the organic solvent employed $[90,91]$. The choice of organic solvent also affects the yield of micellization. For example, La et al. [90] reported that the use of dimethylsulfoxide (DMSO) as the organic solvent which gave rise to PEO- $b$-poly ( $\beta$-benzyl-L-aspartate) (PBLA) micelles which were only $17 \mathrm{~nm}$ in size, but the micelle yield was low (6\%). Using dimethylacetamide (DMAc) as organic solvent resulted in a much higher micelle yield, with an average particle size of $19 \mathrm{~nm}$ and a narrow size distribution [90]. In this way, the dialysis method provides a means of tailoring the size and size distribution of the micelles. Note that, above all, it is important to select conditions that minimize the formation of large polymer aggregates (diameter $>300 \mathrm{~nm}$ ), in addition to the desired polymeric micelles [92].

We determined the size of DEX-based polymeric micelles by dynamic laser light scattering (DLS) at $25{ }^{\circ} \mathrm{C}$, with a scattering angle of $90^{\circ}$, and found that the hydrodynamic diameter of DEX- $g$ - $\mathrm{PEO}_{10}-\mathrm{C}_{16}$ micelles ranged from 10 to $45 \mathrm{~nm}$ (Table 1). In all cases, the size distribution was unimodal, indicative of the absence of free polymer chains and of large aggregates. Polymeric micelles formed by amphiphilic DEXs of structure similar to that of DEX10- $g-\mathrm{PEO}_{y}-\mathrm{C}_{n}$, but prepared from a higher molecular weight DEX (Dextran T40; MW 40000 Da; DEX40) are larger than those formed by DEX10- $g-\mathrm{PEO}_{y}-\mathrm{C}_{n}$, independently of the size of the hydrophobic substituent and of the level of modification [93]. This observation can be taken as an indication of the steric hindrance induced by the carbohydrate chains, which are expected to take place over a larger volume for the polymer of higher molecular weight $[94,95]$. It has been shown that the uptake of particles within the intestine and the extent of drug absorption increase with decreasing particle size and increasing specific surface area [6]. Thus, the small size exhibited by all the polymeric micelles studied here shows a favorable trend toward oral drug delivery. 


\section{PHYSICAL LOADING OF A DRUG IN DEX-g-PEO ${ }_{10}-\mathrm{C}_{16}$ POLYMERIC MICELLES}

The hydrophobic core of the polymeric micelles is expected to serve as the loading space for various lipophilic drugs. By design, given the nanometric size of the micelles, this space is limited. In order to exploit fully this loading space, one must manipulate the many factors that control loading capacity and efficiency. In devising a drug incorporation strategy, one must try to match as closely as possible the polarity of the hydrophobic micelle core to the solubility characteristics of the drug. Nagarajan et al. [96] demonstrated that aromatic hydrocarbons are incorporated to a greater extent into poly(ethylene oxide- $b$-propylene oxide) and poly ( $N$-vinylpyrrolidone- $b$-styrene) polymeric micelles, in comparison to aliphatic hydrocarbons. Kim et al. [91] showed that the amount of indomethacin that can be incorporated into micelles can reach 42.2 (wt $\%$ ) in the case of PEO- $b$-poly ( $\varepsilon$-caprolactone), while La et al. [90] obtained an indomethacin content of only 22.1 (wt \%) for micelles formed from PEO- $b$-PBLA. An increase in the length of the core-forming segment tends to enhance the loading capacity per micelle [97]. Note that a drug may reside preferably within the core of the micelle or within the core/corona interface, depending on its solubility characteristics [97].

We report here the loading capacity of DEX- $g-\mathrm{PEO}_{10}-\mathrm{C}_{16}$ polymeric micelles toward cyclosporin A (CsA), a highly effective immunosuppressive agent that is used for the prevention of graft rejection following organ transplantation [98]. Chemically, CsA is a neutral cyclic undecapeptide with a molecular weight of $1202 \mathrm{~g} / \mathrm{mol}$ [99], consisting of hydrophobic amino acids linked via 11 amide bonds, 7 of which are $N$-methylated. Four intramolecular hydrogen bonds [100] contribute to the rigidity of its skeleton. This chemical composition is responsible for the extremely low solubility of CsA in water $\left(23 \mu \mathrm{g} / \mathrm{ml}\right.$ at $\left.20^{\circ} \mathrm{C}\right)$. The oral administration of CsA is complicated by the presence of several metabolizing enzymes: cytochrome P-450 3A4 (CYP3A4), the multidrug transporter P-glycoprotein (PGP) in the small intestine, and hepatic CYP3A4 [101]. These factors, together with the poor solubility of CsA in the aqueous fluids of the GI tract, severely limit the absorption of CsA through the GI mucosa. They account for the low bioavailability of the drug and significantly increase the risks of both acute and chronic rejection [102]. Commercial CsA oral formulations are mostly microemulsion-based. While more effective than the original emulsion-based formulations, this formulation presents a rather large interindividual variation in terms of CsA bioavailability [103]. In view of the clinical importance of CsA, much effort has been placed toward designing oral formulations leading to acceptable bioavailability. A number of innovative drug delivery approaches including mixed micelles [104], charged nanoparticles [105], liposomes [106], lipids [107], surfactants [108], microspheres [109], and microemulsions [110] have been investigated in order to improve the unfavorable absorption characteristics of CsA.

We chose to incorporate CsA into DEX- $g-\mathrm{PEO}_{10}-\mathrm{C}_{16}$ polymeric micelles by a dialysis method, which involved treatment of an aqueous polymer solution with a solution of CsA in ethanol. Ethanol was used as solvent to prevent any cytotoxic effect that could be caused by trace organic solvent in the final formulation. The initial CsA loading varied from 2.5-40\% ( $w / w$ CsA/polymer). The mixed solutions were extensively dialyzed against distilled water. In this process, the organic solvent is slowly replaced by water so that micelles start to form and to incorporate the lipophilic drug inside their hydrophobic core (Fig. 5). After completion of the dialysis, the solutions were filtered to eliminate excess CsA and the filtrate was freeze-dried, yielding a free-flowing powder which readily dispersed in water to form polymeric micelles of size and size distribution nearly identical to those of micelles devoid of CsA. 


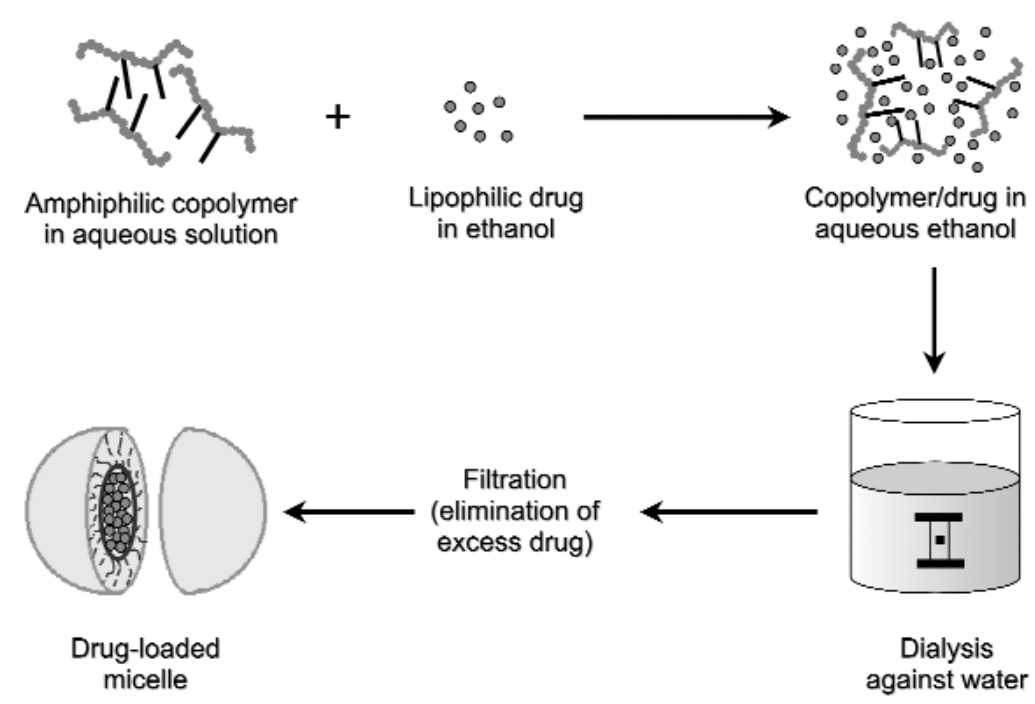

Fig. 5 Schematic representation of drug loading in polymeric micelles using the dialysis method.

The amount of CsA incorporated in the micelles was determined by a high-performance liquid chromatography quantitative assay of the CsA extracted from freeze-dried micelles with acetonitrile, a nonsolvent of the copolymer, but a good solvent of CsA. Drug loading (DL) was calculated using eq. 1:

$$
\operatorname{DL}(\%)=100\left(W_{\mathrm{c}} / W_{\mathrm{M}}\right)
$$

where $W_{\mathrm{c}}$ is the weight of CsA loaded in micelles and $W_{\mathrm{M}}$ is the weight of micelles before extraction. The amount of CsA released upon dissolution was plotted against the CsA concentration added initially for each preparation. Both free and micelle-entrapped CsA were measured with this assay. The amount of free CsA is expected to be low, since the undissolved fraction of the drug should have been removed by filtration after dialysis.

We evaluated first the ability of the $\mathrm{PEO}_{10}-\mathrm{C}_{16}$ micelles to solubilize CsA and found that a high level of CsA $(17.5 \% \mathrm{w} / \mathrm{w})$ was incorporated in the surfactant micelles, an indication of the favorable solubilizing characteristics of $\mathrm{PEO}_{10} \mathrm{C}_{16}$ toward CsA. Next, we assessed the incorporation of CsA within polymeric micelles. The maximum amount of CsA loaded within the micelles was 1,7 , and $8 \%$ (w/w), for DEX-g-PEO ${ }_{10}-\mathrm{C}_{16}$ containing 2.3, 6, and $15 \mathrm{~mol} \% \mathrm{PEO}_{10}-\mathrm{C}_{16}$, respectively (Fig. 6). In all cases, the amount of CsA incorporated was larger in the case of polymeric micelles than in the case of unmodified DEX, which proved to have a very low affinity for CsA. By comparing the highest CsA loading percentage in the various polymers, it is apparent that the CsA loading increases with increasing molar content of $\mathrm{PEO}_{10}-\mathrm{C}_{16}$ grafted on the DEX backbone. Other structural features of the copolymers also affect the micellar loading capacity toward CsA. For example, micelles formed by polymers of higher molecular weights (e.g., DEX40) are not as effective CsA carriers as those based on DEX10 [93]. Also, for a constant number of $\mathrm{PEO}_{10}-\mathrm{C}_{n}$ units grafted per DEX chain, $\mathrm{PEO}_{10}-\mathrm{C}_{16}$-based micelles seem more effective in solubilizing CsA than $\mathrm{PEO}_{10}-\mathrm{C}_{18}$-based micelles, indicating that the micropolarity of a hydrophobic core made up of hexadecyl-PEO chains might present a better solubilizing microenvironment than that offered by the octadecyl-PEO moieties [93]. 


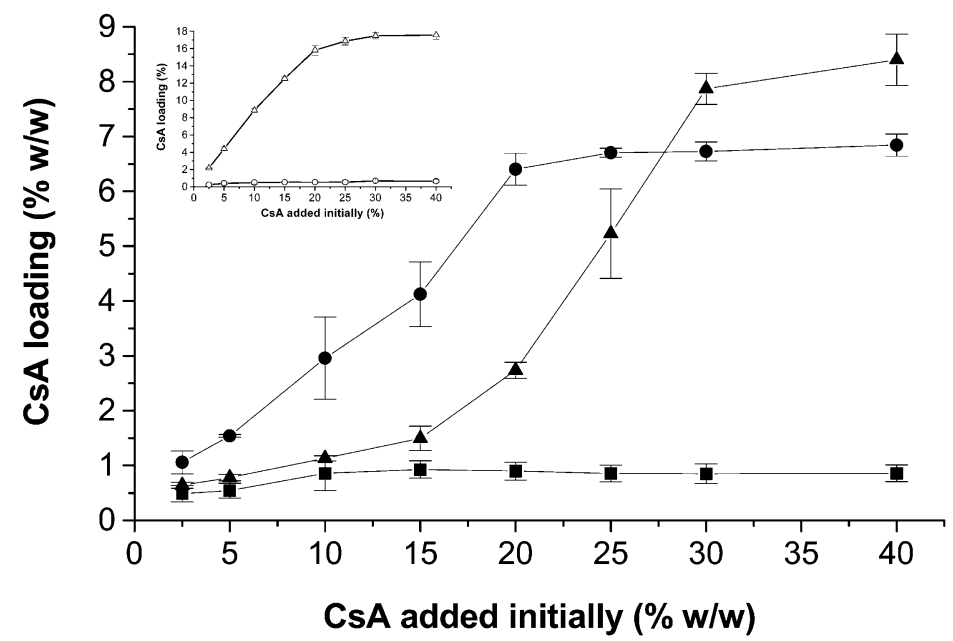

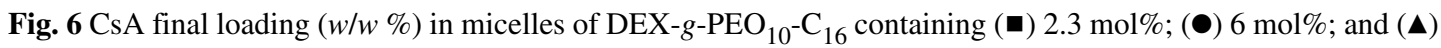
$15 \mathrm{~mol} \%$ grafted $\mathrm{PEO}_{10}-\mathrm{C}_{16}$ residues, at 2.5-40 (\% w/w) of initially added CsA. Inset: CsA loading (\% w/w) in presence of $(\triangle)$ free $\mathrm{PEO}_{10}-\mathrm{C}_{16}$ surfactant as well as $(\bigcirc)$ unmodified DEX polymer. Mean $\pm \operatorname{SD}(n=3)$.

\section{CYTOTOXICITY STUDY}

The human colon adenocarcinoma, Caco- 2 cells, are generally selected to estimate in vivo drug absorption, as they retain many features of small intestinal epithelial cells [111,112]. Thus, we used Caco-2 cells to study the cytotoxicity of DEX- $g$ - $\mathrm{PEO}_{10}-\mathrm{C}_{16}$ polymeric micelles, using the colorimetric MTT assay [113]. The cell viability in the presence of micelles was compared to that in the presence of unmodified DEX, as well as of free $\mathrm{PEO}_{10}-\mathrm{C}_{16}$ surfactant residues (Fig. 7).

Cell growth was severely inhibited by $\mathrm{PEO}_{10}-\mathrm{C}_{16}$, even at surfactant concentrations below $1 \mathrm{~g} / \mathrm{l}$. Such deleterious effect is consistent with the reported propensity of PEO alkyl ethers to affect membrane integrity [114]. In contrast, the polysaccharide DEX showed no toxicity up to a concentration of

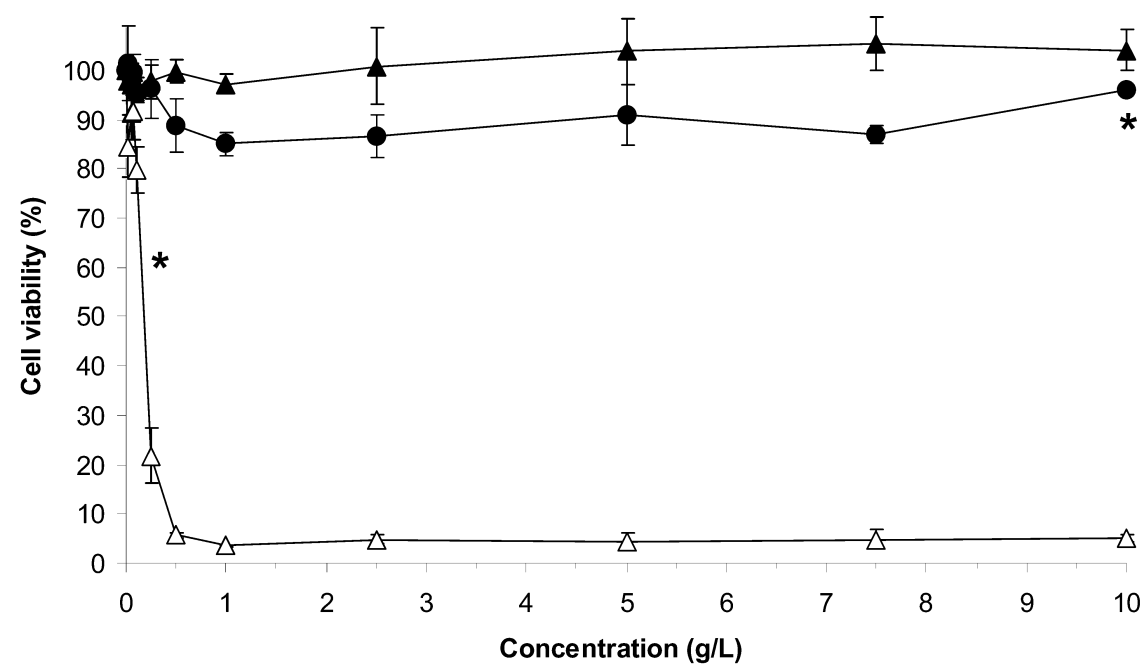

Fig. 7 Effect of unmodified DEX $(\Delta)$, free $\mathrm{PEO}_{10}-\mathrm{C}_{16}(\triangle)$ and DEX-g-PEO${ }_{10}-\mathrm{C}_{16}(15 \mathrm{~mol} \%)(\bullet)$ on Caco-2 cell viability measured by the MTT assay. Equivalent concentrations of free $\mathrm{PEO}_{10}-\mathrm{C}_{16}$ and $\mathrm{PEO}_{10}-\mathrm{C}_{16}$ grafted to DEX backbone are indicated in the figure by stars. Mean $\pm \operatorname{SD}(n=3)$. 
$10 \mathrm{~g} / \mathrm{l}$, confirming the reported inertness of DEXs. Turning now to the effect of DEX- $g$-PEO $10^{-} \mathrm{C}_{16}$ (15 mol\%), we determined that it exhibited no significant toxicity toward Caco-2 cells, up to concentrations of $10 \mathrm{~g} / \mathrm{l}$ or concentration of $\mathrm{PEO}_{10}-\mathrm{C}_{16}(0.05 \mathrm{~g} / \mathrm{l})$ equivalent to a $\mathrm{PEO}_{10}-\mathrm{C}_{16}$ concentration showing a significant cytototoxic effect (60\% cell viability). This important result indicates (1) that the polymer purification method efficiently removed any free $\mathrm{PEO}_{10}-\mathrm{C}_{16}$ from the polymer, and (2) by grafting the $\mathrm{PEO}_{10}-\mathrm{C}_{10}$ residues onto DEX, we succeeded in minimizing their toxic effect on cells. The nontoxic hydrophilic DEX chains forming the polymeric micelle outer shell may be in contact with the cells and effectively insulate them from the surfactant residues assembled in the inner core.

\section{CONCLUSION}

The experimental evidence reviewed in this article led us to conclude that polymeric micelles present a number of enabling properties for oral delivery of lipophilic drugs. For example, HM DEX forms nanosized assemblies characterized by a low onset of micellization and a high solubilization power toward highly lipophilic drugs. Moreover, they present no significant cytotoxicity. Initial results indicate that the transport of an HM DEX-entrapped lipophobic drug is enhanced, compared to a free drug [115]. Current research on particulate delivery systems is focused on gaining a better understanding of the mechanisms, efficiency, and reproducibility of the permeability of both the carrier and/or the drugs across the GI tract, and analysis of the effect of carrier composition on these parameters.

\section{ACKNOWLEDGMENTS}

This work was supported financially by the Natural Sciences and Engineering Research Council of Canada under its strategic grants program. M. F. Francis acknowledges a scholarship from the Rx\&D Health Research Foundation (HRF)/Canadian Institutes of Health Research (CIHR).

\section{REFERENCES}

1. E. C. Lavelle, S. Sharif, N. W. Thomas, J. Holland, S. S. Davis. Adv. Drug Delivery Rev. 18, 5-22 (1995).

2. A. L. Daugherty and R. J. Mrsny. Pharm. Sci. Technol. Today 2, 144-151 (1999).

3. D. Horter and J. B. Dressman. Adv. Drug Delivery Rev. 46, 75-87 (2001).

4. W. N. Charman and V. J. Stella. Adv. Drug Delivery Rev. 7, 1-14 (1991).

5. H. Chen and R. Langer. Adv. Drug Delivery Rev. 34, 339-350 (1998).

6. A. T. Florence and N. Hussain. Adv. Drug Delivery Rev. 50, S69-S89 (2001).

7. W. Sass, H. P. Dreyer, J. Seifert. Am. J. Gastroenterol. 85, 255-260 (1990).

8. P. G. Jenkins, K. A. Howard, N. W. Blackball, N. W. Thomas, S. S. Davis, D. T. O'Hagan. J. Controlled Release 29, 339-350 (1994).

9. P. Jani, G. W. Halbert, J. Langridge, A. T. Florence. J. Pharm. Pharmacol. 41, 809-812 (1989).

10. P. Jani, G. W. Halbert, J. Langridge, A. T. Florence. J. Pharm. Pharmacol. 42, 821-826 (1990).

11. M. P. Desai, V. Labhasetwar, G. L. Amidon, R. J. Levy. Pharm. Res. 13, 1838-1845 (1996).

12. J. H. Eldridge, C. J. Hammond, A. Meulbroek, J. K. Staas, R. M. Gilley, T. R. Tice. J. Controlled Release 11, 205-214 (1990).

13. A. M. Hillery, P. U. Jani, A. T. Florence. J. Drug Target. 2, 151-156 (1994).

14. E. Mathiowitz, J. S. Jacob, Y. S. Jong, G. P. Carino, D. Chickering, P. Charturved, C. A. Santos, K. Vijayaraghavan, S. Montogomery, M. Bassett, C. Morrell. Nature 386, 410-414 (1997).

15. B. Kriwet, E. Walter, T. Kissel. J. Controlled Release 56, 149-158 (1998).

16. C. Damgè, C. Michel, M. Aprahamian, P. Couvreur. Diabetes 37, 246-251 (1988).

17. A. Sharma and U. S. Sharma. Int. J. Pharm. 154, 123-140 (1997).

18. J. Okada, S. Cohen, R. Langer. Pharm. Res. 12, 576-582 (1995). 
19. D. S. Deshmukh, W. D. Bear, H. Brockerhoff. Life Sci. 28, 239-242 (1980).

20. E. Toorisaka, H. Ono, K. Arimori, N. Kamiya, M. Goto. Int. J. Pharm. 252, 271-274 (2003).

21. K. Itoh, S. Matsui, Y. Tozuka, T. Oguchi, K. Yamamoto. Int. J. Pharm. 246, 75-83 (2002).

22. P. Vischer and J. Casals-Stenzel. J. Pharm. Pharmacol. 35, 152-156 (1983).

23. M. Uchida, Y. Kato, K. Matsueda, R. Shoda, A. Muraoka, S. Yamato. Jpn. J. Pharmacol. 82, 168-170 (2000).

24. P. Couvreur and F. Puisieux. Adv. Drug Delivery Rev. 10, 141-162 (1993).

25. S. Sakuma, M. Hayashi, M. Akashi. Adv. Drug Delivery Rev. 47, 21-37 (2001).

26. T. Jung, W. Kamm, A. Breitenbach, E. Kaiserling, J. X. Xiao, T. Kissel. Eur. J. Pharm. Biopharm. 50, 147-160 (2000).

27. P. Sai, C. Damage, A. S. Rivereau, A. Hoeltzel, E. Gouin. J. Autoimmun. 9, 713-722 (1996).

28. M. Saffran, G. S. Kumar, C. Savariar, J. C. Burnham, F. Williams, D. C. Neckers. Science 233, 1081-1084 (1986).

29. P. Edman, B. Ekman, I. Sjoholm. J. Pharm. Sci. 69, 838-842 (1980).

30. A. K. Andrianov and L. G. Payne. Adv. Drug Delivery Rev. 34, 155-170 (1998).

31. K. Matsuno, T. Schaffner, H. A. Gerber, C. Ruchti, M. W. Hess, H. Cottier. J. Reticuloendothel. Soc. 33, 263-273 (1983).

32. M. Kukan, V. Koprda, S. Bezek, J. Kalal, J. Labsky, T. Trnovec. Pharmazie 46, 37-39 (1991).

33. J. Kreuter. Adv. Drug Delivery Rev. 7, 71-86 (1991).

34. A. Fasano. Trends Biotechnol. 16, 152-157 (1998).

35. T. Gershanik and S. Benita. Eur. J. Pharm. Biopharm. 50, 179-188 (2000).

36. C. Leuner and J. Dressman. Eur. J. Pharm. Biopharm. 50, 47-60 (2000).

37. J. K. Vasir, K. Tambwekar, S. Garg. Int. J. Pharm. 255, 13-32 (2003).

38. M. Yokoyama, G. S. Kwon, T. Okano, Y. Sakurai, T. Seto, K. Kataoka. Bioconjugate Chem. 3, 295-301 (1992).

39. T. S. Wiedmann and L. Kamel. J. Pharm. Sci. 91, 1743-1764 (2002).

40. K. Kataoka, G. S. Kwon, M. Yokoyama, T. Okano, Y. Sakurai. J. Controlled Release 24, 119-132 (1993).

41. M. Yokoyama, M. Miyauchi, N. Yamada, T. Okano, Y. Sakurai, K. Kataoka, S. Inoue. Cancer Res. 50, 1693-1700 (1990).

42. V. H. L. Lee and A. Yamamoto. Adv. Drug Delivery Rev. 4, 171-207 (1990).

43. V. P. Torchilin. J. Controlled Release 73, 137-172 (2001).

44. K. Kataoka. J. Macromol. Sci., Pure Appl. Chem. A31, 1759-1769 (1994).

45. M. Yokoyama (Ed.). Novel Passive Targetable Drug Delivery with Polymeric Micelles, pp. 193-229, Academic Press, San Diego (1998).

46. S. N. Malik, D. H. Canaham, M. W. Gouda. J. Pharm. Sci. 64, 987-990 (1975).

47. K. Takada, H. Yoshimura, N. Shibata, Y. Masuda, H. Yoshikawa, S. Muranishi, T. Yasumura, T. Oka. J. Pharmacobio-Dyn. 9, 156-160 (1986).

48. D. D. Lasic. Nature 355, 279-280 (1992).

49. G. Schubiger, J. Gruter, M. J. Shearer. J. Pediatr. Gastroenterol. Nutr. 24, 280-284 (1997).

50. P. A. Redondo, A. I. Alvarez, J. L. Garcia, C. Villaverde, J. G. Prieto. Biopharm. Drug Dispos. 19, 65-70 (1998).

51. H. A. Bardelmeijer, M. Ouwehand, M. M. Malingre, J. H. Schellens, J. H. Beijnen, O. van Tellingen. Cancer Chemother. Pharmacol. 49, 119-125 (2002).

52. D. L. Elbert and J. A. Hubbell. Chem. Biol. 5, 177-183 (1998).

53. H. Otsuka, Y. Nagasaki, K. Kataoka. Curr. Opin. Colloid Interface Sci. 6, 3-10 (2001).

54. H. Otsuka, Y. Nagasaki, K. Kataoka. Adv. Drug Delivery Rev. 55, $403-419$ (2003).

55. G. Kwon, S. Suwa, M. Yokoyama, T. Okano, Y. Sakurai, K. Kataoka. J. Controlled Release 29, 17-23 (1994).

56. G. S. Kwon and K. Kataoka. Adv. Drug Delivery Rev. 16, 295-309 (1995).

(C) 2004 IUPAC, Pure and Applied Chemistry 76, 1321-1335 
57. Y. Yamamoto, Y. Nagasaki, Y. Kato, Y. Sugiyama, K. Kataoka. J. Controlled Release 77, 27-38 (2001).

58. G. S. Kwon and T. Okano. Adv. Drug Delivery Rev. 21, 107-116 (1996).

59. K. Kataoka, A. Harada, Y. Nagasaki. Adv. Drug Delivery Rev. 47, 113-131 (2001).

60. G. S. Kwon. Adv. Drug Delivery Rev. 54, 167 (2002).

61. X. Zhang, H. M. Burt, G. Mangold, D. Dexter, D. Von Hoff, L. Mayer, W. L. Hunter. Anticancer Drugs 8, 696-701 (1997).

62. I. G. Shin, S. Y. Kim, Y. M. Lee, C. S. Cho, Y. K. Sung. J. Controlled Release 51, 1-11 (1998).

63. B. G. Yu, T. Okano, K. Kataoka, S. Sardari, G. S. Kwon. J. Controlled Release 56, 285-291 (1998).

64. Y. I. Jeong, J. W. Nah, H. C. Lee, S. H. Kim, C. S. Cho. Int. J. Pharm. 188, 49-58 (1999).

65. C. Allen, J. Han, Y. Yu, D. Maysinger, A. Eisenberg. J. Controlled Release 63, 275-286 (2000).

66. E. V. Batrakova, H. Y. Han, V. Y. Alakhov, D. W. Miller, A. V. Kabanov. Pharm. Res. 15, 850-855 (1998).

67. E. V. Batrakova, H. Y. Han, D. W. Miller, A. V. Kabanov. Pharm. Res. 15, 1525-1532 (1998).

68. E. V. Batrakova, S. Li, D. W. Miller, A. Kabanov. Pharm. Res. 16, 1366-1372 (1999).

69. V. Alakhov, E. Klinski, P. Lemieux, G. Pietrzynski, A. Kabanov. Expert. Opin. Biol. Ther. 1, 583-602 (2001).

70. A. Kabanov, E. Batrakova, V. Alakhov. J. Controlled Release 82, 189-212 (2002).

71. A. V. Kabanov and V. Y. Alakhov. Crit. Rev. Ther. Drug Carrier Syst. 19, 1-72 (2002).

72. P. Lemieux, S. V. Vinogradov, C. L. Gebhart, N. Guerin, G. Paradis, H. K. Nguyen, B. Ochietti, Y. G. Suzdaltseva, E. V. Bartakova, T. K. Bronich, Y. St-Pierre, V. Y. Alakhov, A. V. Kabanov. J. Drug Target. 8, 91-105 (2000).

73. C. Larsen. Adv. Drug Delivery Rev. 3, 103-154 (1989).

74. N. P. Couch. Clin. Pharmacol. Ther. 6, 656-665 (1965).

75. Y. Machida and T. Nagai. Chem. Pharm. Bull. 22, 2346-2351 (1974).

76. G. W. Skinner, W. W. Harcum, P. E. Barnum, J. H. Guo. Drug. Dev. Ind. Pharm. 25, 1121-1128 (1999).

77. M. Cristea and F. M. Winnik. Macromolecules (2004). Submitted for publication.

78. R. Nagarajan and K. Ganesh. Macromolecules 22, 4312-4325 (1989).

79. R. Zana. Adv. Colloid Interface Sci. 97, 205-253 (2002).

80. P. Alexandridis, J. F. Holzwarth, T. A. Hatton. Macromolecules 27, 2414-2425 (1994).

81. I. Astafieva, Z. F. Zhong, A. Eisenberg. Macromolecules 29, 7339-7352 (1993).

82. P. Alexandridis, V. Athanassiou, S. Fukuda, T. A. Hatton. Langmuir 10, 2604-2612 (1994).

83. D. C. Dong and M. A. Winnik. Can. J. Chem. 62, 2560-2565 (1984).

84. C. L. Zhao, M. A. Winnik, G. Riess, M. D. Croucher. Langmuir 6, 514-516 (1990).

85. F. M. Winnik and S. T. A. Regismond. In Polymer-Surfactant Systems, I. C. T. Kwak (Ed.), pp. 267-315, Marcel Dekker, New York (1998).

86. K. Kalyanasundaram and J. K. Thomas. J. Am. Chem. Soc. 99, 2039-2044 (1977)

87. M. Wilhelm, C. L. Zhao, Y. Wang, R. Xu, M. A. Winnik, J. L. Mura, G. Riess, M. D. Croucher. Macromolecules 24, 1033-1040 (1991).

88. A. Halperin. Macromolecules 20, 2943-2946 (1987).

89. R. Nagarajan and K. Ganesh. J. Chem. Phys. 90, 5843-5856 (1989).

90. S. B. La, T. Okano, K. Kataoka. J. Pharm. Sci. 85, 85-90 (1996).

91. S. Y. Kim, I. G. Shin, Y. M. Lee, C. S. Cho, Y. K. Sung. J. Controlled Release 51, 13-22 (1998).

92. S. Cammas, K. Suzuki, C. Sone, Y. Sakurai, K. Kataoka, T. Okano. J. Controlled Release 48, 157-164 (1997).

93. M. F. Francis, L. Lavoie, F. M. Winnik, J. C. Leroux. Eur. J. Pharm. Biopharm. 56, 337-346 (2003). 
94. A. V. Maksimenko, Y. V. Schechilina, E. G. Tischenko. Biochemistry (Moscow) 66, 456-463 (2001).

95. A. L. Baldwin and S. Chien. Arteriosclerosis 8, 140-146 (1988).

96. R. Nagarajan, M. Barry, E. Ruckenstein. Langmuir 2, 210-215 (1986).

97. F. Gadelle, W. J. Koros, R. S. Schechter. Macromolecules 28, 4883-4892 (1995).

98. A. Laupacis, P. Keown, R. Ulan, N. McKenzie, C. Stiller. Can. Med. Assoc. J. 126, 1041-1046 (1982).

99. R. Wenger. Transplant. Proc. 15, 2230-2241 (1983).

100. W. Vine and L. Bowers. Crit. Rev. Clin. Lab. Sci. 25, 275-311 (1987).

101. J. F. Tjia, I. R. Webber, D. J. Back. Br. J. Clin. Pharmacol. 31, 344-346 (1991).

102. A. Lindholm, S. Henricsson, M. Lind, R. Dahlqvist. Eur. J. Clin. Pharmacol. 34, 461-464 (1988).

103. J. M. Kovarik, E. A. Mueller, J. B. van Bree, S. S. Fluckiger, H. Lange, B. Schmidt, W. H. Boesken, A. E. Lison, K. Kutz. Transplantation 58, 658-663 (1994).

104. K. Takada, N. Shibata, H. Yoshimura, Y. Masuda, H. Yoshikawa, S. Muranishi, T. Oka. J. Pharmacobio-Dyn. 8, 320-323 (1985).

105. M. H. El-Shabouri. Int. J. Pharm. 249, 101-108 (2002).

106. M. Al-Meshal, S. H. Khidr, M. A. Bayomi, A. A. Al-Angary. Int. J. Pharm. 168, 163-168 (1998).

107. C. T. Ueda, M. Lemaire, G. Gsell, K. Nussbaumer. Biopharm. Drug Dispos. 4, 113-124 (1983).

108. T. Chang, L. Z. Benet, M. F. Hebert. Clin. Pharmacol. Ther. 59, 297-303 (1996).

109. S. J. Kim, H. K. Choi, S. P. Suh, Y. B. Lee. Eur. J. Pharm. Sci. 15, 497-502 (2002).

110. W. A. Ritschel, D. G. Patel, P. Chalasani, T. Schroeder. Pharm. Res. 7, 5-119 (1990).

111. A. H. Dantzig and L. Bergin. Biochim. Biophys. Acta 1027, 211-217 (1990).

112. I. J. Hidalgo, T. J. Raub, R. T. Borchardt. Gastroenterology 96, 736-749 (1989).

113. M. B. Hansen, S. E. Nielsen, K. Berg. J. Immunol. Methods 119, 203-210 (1989).

114. D. Dimitrijevic, A. J. Shaw, A. T. Florence. J. Pharm. Pharmacol. 52, 157-162 (2000).

115. M. F. Francis, M. Cristea, F. M. Winnik, J. C. Leroux. Proceed. Intern. Symp. Control. Rel. Bioact. Mater. 30, 68-69 (2003). 